\title{
Magnetic-order-induced ferroelectricity in orthorhombic perovskite manganites
}

\author{
C. D. $\mathrm{Hu}$ \\ Department of Physics and Center for Theoretical Science, National Taiwan University, Taipei 10617, Taiwan, Republic of China
}

(Received 19 April 2007; revised manuscript received 30 April 2007; published 30 May 2007)

\begin{abstract}
We studied the ferroelectricity of magnetic oxides, in which its emergence coincides with the onset of a second incommensurate magnetic order. We solved for the wave function of $e_{g}$ electrons in the presence of magnetic orders. The coupling between the magnetic and electric orders is provided by the spin-orbit interaction. It was found that the net electric dipole moment of the system came from the bond between the transition metal and oxygen atoms. The anisotropy of $d$ orbitals also played an important role. Finally, the form of the coupling leads us to the conclusion that it is an improper ferroelectricity.
\end{abstract}

DOI: 10.1103/PhysRevB.75.172106

The multiferroic phenomena have been studied for a long time. ${ }^{1}$ The interest has been revived by recent experimental findings. They showed that magnetic and ferroelectric orders are closely related. ${ }^{2-5}$ For example, $\mathrm{TbMnO}_{3}$ undergoes antiferromagnetic phase transition at $41 \mathrm{~K}$, at which an incommensurate sinusoidal magnetic order begins to develop. Below $28 \mathrm{~K}$, which was often referred to as $T_{\text {lock }}$, a second magnetic order was observed. ${ }^{6}$ This occurrence coincides with the divergence of dielectric constant and the emergence of the electric dipole moment of the system. It is this fascinating interplay between ferroelectric and magnetic orders that has attracted many researchers. As far as we know, the mechanism of the coupling between the incommensurate magnetic orders and ferroelectricity is still an unsolved topic. There have been calculations of electric polarization based on atomic displacements or lattice distortions. ${ }^{7,8}$ There are also proposals of dipole moment due to electronic distribution..$^{9,10}$ However, it is interesting to note that the spin-orbit interaction played a crucial role in both schools. Sergienko and Dagotto ${ }^{8}$ espoused the idea that ferroelectricity is produced by Dzyaloshinskii-Moriya (DM) interaction. ${ }^{11,12}$ Katsura et al. ${ }^{10}$ showed that magnetoelectric effect was induced by spin current via spin-orbit coupling. In Refs. 8 and 9, phase diagrams were drawn and the regions of ferroelectricity were identified. However, it is still not clear how magnetic orders are coupled to ferroelectricity. If it is due to DM interaction, then the question remains as to what the driving force is. Is it from the displacement of atoms as was suggested in Ref. 8? If so, why is there a linear relation between the displacement and the coupling constant $\boldsymbol{D}$ ? Does it come from an "internal electric field." as was proposed in Ref. 10? A theory of spin current coupling to the motion of charge is presented. The computation is based on only two noncollinear spins. Then, the question arises as to how the mechanism works in a collective manner, i.e., in a crystal.

In this work, we tried to give a global view. We proposed a possible mechanism of coupling between the magnetic orders and the ferroelectric order. The systems we had in mind were orthorhombic perovskites such as $\mathrm{TbMnO}_{3}$. These are complex systems in which Hund's coupling, anisotropy of orbitals, and lattice distortion all have significant effect. The spin-orbit interaction also played a subtle role. Thus, our Hamiltonian has the form $H=H_{0}+H_{1}$, with the latter being the spin-orbit interaction and
PACS number(s): 77.80.-e, 75.80.+q

$$
\begin{aligned}
H_{0}= & \sum \varepsilon_{p} c_{i, l p \sigma}^{\dagger} c_{i, l p \sigma}+\sum \varepsilon_{d} c_{j, d \sigma}^{\dagger} c_{j, d \sigma} \\
& +\sum t_{l, i j}\left(c_{i, l p \sigma}^{\dagger} c_{j, d \sigma}+c_{j, d \sigma}^{\dagger} c_{i, l p \sigma}\right)-J \sum S_{j} \cdot s_{j} .
\end{aligned}
$$

Here, $\varepsilon_{p}, \varepsilon_{d}, c_{i, l p \sigma}$, and $c_{j, d \sigma}$ are the energies and field operators of the electrons in the $p$ orbitals in oxygen and $d$ orbitals in transition element ions, with $\sigma$ being the spin index and $l$ the oxygen-atom index in the basis. To simplify the computation, we considered the dynamics on the $a b$ plane. We also made the approximation that the lattice constants $a$ and $b$ are equal. As a result, there is one transition element atom and two oxygen atoms in our basis. Let one metal $(M)-\mathrm{O}$ $(l=x)$ bond be along the $x$ direction and the other $(l=y)$ along the $y$ direction. Thus the oxygen atoms are at $\boldsymbol{R}_{j}+\boldsymbol{r}_{x}$ and $\boldsymbol{R}_{j}+\boldsymbol{r}_{y}$ in the $j$ th unit cell, where $\boldsymbol{r}_{x}=\hat{x} b / 2 \sqrt{2}$ and $\boldsymbol{r}_{y}$ $=\hat{y} b / 2 \sqrt{2}$, with $b / \sqrt{2}$ being our reduced lattice constant. The orbitals we chose were the most active ones. For example, the $p_{x}$ orbitals of oxygen atoms were incorporated if the $M-\mathrm{O}$ bond was along the $x$ direction, and $p_{y}$ orbitals for $y$-direction bonds. For the same reason, we considered only one $e_{g}$ orbital. There are two $e_{g}$ orbitals. Due to the Jahn-Teller lattice distortion, the degeneracy is lifted. The lower state (as well as the upper) has the form $A\left|x^{2}-y^{2}\right\rangle+B\left|3 z^{2}-r^{2}\right\rangle$ and this is the state we considered. We later found out that the values of $A$ and $B$ were not going to qualitatively affect our results. The tight-binding matrix elements $t_{l, i j}$ can be found in Slater and Koster: ${ }^{13}$ $t_{l, i j}=(-1)^{i-j}\left(\Lambda_{l} A+B / \sqrt{3}\right)(p d \sigma)$, where $\Lambda_{l}=-1$ (1) if $l=x(y)$. The fourth term is Hund's coupling. $S_{j}$ and $\boldsymbol{s}_{j}$ denote the spins of the $t_{2 g}$ and $e_{g}$ electrons. The $t_{2 g}$ electrons of metal ions are relatively inert since their energy is several $\mathrm{eV}$ lower than that of $e_{g}$ electrons. They form an $S=3 / 2$ state. Their effect is to provide a fictitious magnetic field to align the spins of $e_{g}$ electrons.

From the start, we assume that there is a spiral magnetic order due to the $t_{2 g}$ electrons. Neutron-scattering data ${ }^{6}$ indicate that this is, indeed, this case below $T_{\text {lock }}$. The spins of $t_{2 g}$ electrons can be expressed as

$$
\begin{gathered}
S_{j}^{x}=S_{0}^{x} \cos \left(\boldsymbol{q} \cdot \boldsymbol{R}_{j}+\gamma\right), \\
S_{j}^{y}=S_{0}^{y} \cos \left(\boldsymbol{q} \cdot \boldsymbol{R}_{j}+\gamma\right), \\
S_{j}^{z}=S_{0}^{z} \cos \left(\boldsymbol{q} \cdot \boldsymbol{R}_{j}\right),
\end{gathered}
$$


where $\boldsymbol{q}$ is the wave vector of the incommensurate order and $\gamma$ is the phase difference between the oscillations of $S^{z}$ and $S^{x(y)}$.

We can then take the Fourier transform of the Hamiltonian in Eq. (1) and reach

$$
\begin{aligned}
H_{0}= & \sum \varepsilon_{p} c_{\mathbf{k}, l p \sigma}^{\dagger} c_{\mathbf{k}, l p \sigma}+\sum \varepsilon_{d} c_{\mathbf{k}, d \sigma}^{\dagger} c_{\mathbf{k}, d \sigma}+\sum\left(V_{\mathbf{k}}^{l} c_{\mathbf{k}, l p \sigma}^{\dagger} c_{\mathbf{k}, d \sigma}+V_{\mathbf{k}}^{l^{*}} c_{\mathbf{k}, d \sigma}^{\dagger} c_{\mathbf{k}, l p \sigma}\right) \\
& -\left(\frac{J}{2}\right) \sum\left[S_{0}^{z}\left(c_{\mathbf{k}, d \uparrow}^{\dagger} c_{\mathbf{k}+\mathbf{q}, d \uparrow}-c_{\mathbf{k}, d \downarrow}^{\dagger} c_{\mathbf{k}+\mathbf{q}, d \downarrow}\right)+S_{0}^{+} e^{i \gamma} c_{\mathbf{k}, d \downarrow}^{\dagger} c_{\mathbf{k}+\mathbf{q}, d \uparrow}+S_{0}^{-} e^{i \gamma} c_{\mathbf{k}, d \uparrow}^{\dagger} c_{\mathbf{k}+\mathbf{q}, d \downarrow}+\text { H.c. }\right],
\end{aligned}
$$

where $S_{0}^{ \pm}=S_{0}^{x} \pm i S_{0}^{y}$ and $V_{\mathbf{k}}^{l}=\left(\Lambda_{l} A+B / \sqrt{3}\right)\left(1-e^{i \mathbf{k} \cdot \mathbf{r}_{l}}\right)(p d \sigma)$ with $l=x(y)$. Thus, we have a Hamiltonian similar to that of spindensity wave. There are 12 coupled states, denoted by $c_{\mathbf{k}, d \sigma}, c_{\mathbf{k}+\mathbf{q}, d \sigma}, c_{\mathbf{k}, l p \sigma}$, and $c_{\mathbf{k}+\mathbf{q}, l p \sigma}$. With these 12 states as basis, we wrote the Hamiltonian as a $12 \times 12$ matrix:

$$
\begin{aligned}
& \left.\begin{array}{cccc}
\varepsilon_{d} & 0 & -J S_{0}^{z} / 2 & -J^{\prime *} S_{0}^{-} / 2 \\
0 & \varepsilon_{d} & -J^{\prime} S_{0}^{+} / 2 & J S_{0}^{z} / 2 \\
-J S_{0}^{z} / 2 & -J^{\prime} S_{0}^{-} / 2 & \varepsilon_{d} & 0 \\
-J^{\prime} S_{0}^{+} / 2 & J S_{0}^{z} / 2 & 0 & \varepsilon_{d} \\
V_{\mathbf{k}}^{x} & 0 & 0 & 0 \\
0 & V_{\mathbf{k}}^{x} & 0 & 0 \\
V_{\mathbf{k}}^{y} & 0 & 0 & 0 \\
0 & V_{\mathbf{k}}^{y} & 0 & 0 \\
0 & 0 & V_{\mathbf{k}+\mathbf{q}}^{x} & 0 \\
0 & 0 & V_{\mathbf{k}+\mathbf{q}}^{x} \\
0 & 0 & V_{\mathbf{k}+\mathbf{q}}^{y} & 0 \\
0 & 0 & V_{\mathbf{k}+\mathbf{q}}^{y} \\
{ }_{0} & 0
\end{array}\right] \\
& =\left[\left(E-\varepsilon_{p}\right) J / 2\right]^{2}\left(S_{0}^{z} \mp i \sqrt{S_{0}^{+} S_{0}^{-}} e^{i \gamma}\right)\left(S_{0}^{z} \pm i \sqrt{S_{0}^{+} S_{0}^{-}} e^{-i \gamma}\right) \text {. }
\end{aligned}
$$

The roots can be found analytically. However, their expressions are rather lengthy. It is sufficient for our purpose to consider the approximate forms by using the fact that $(p d \sigma) \ll\left|\varepsilon_{d}-J / 2-\varepsilon_{p}\right|$. Due to the spin-density wavelike interaction, the states $\mathbf{k}$ and $\mathbf{k}+\mathbf{q}$ are coupled. The eigenfunction is a linear combination of these states. Hence, the top two bands are empty if there are five valence electrons (two $p$ electrons for each oxygen atom and one $e_{g}$ electron) in a unit cell. The highest occupied state has the eigenvalue

$$
E_{\mathbf{k}} \simeq \varepsilon_{d}-\frac{J|\Sigma|}{2}+\frac{\Sigma_{l}\left(\left|V_{\mathbf{k}}^{l}\right|^{2}+\left|V_{\mathbf{k}+\mathbf{q}}^{l}\right|^{2}\right)}{2\left(\varepsilon_{d}-J|\Sigma| / 2-\varepsilon_{p}\right)},
$$

where $\Sigma=S_{0}^{z}+i \sqrt{S_{0}^{+} S_{0}^{-}} e^{i \gamma}$. The eigenstate is

$$
\begin{aligned}
\left|\Psi_{\mathbf{k}}\right\rangle= & C \sum_{\sigma}\left[\alpha_{\sigma} c_{\mathbf{k}, d \sigma}^{\dagger}+\beta_{\sigma} c_{\mathbf{k}+\mathbf{q}, d \sigma}^{\dagger}\right. \\
& \left.+\sum_{l} \frac{\left(V_{\mathbf{k}}^{l} \alpha_{\sigma} c_{\mathbf{k}, l p \sigma}^{\dagger}+V_{\mathbf{k}+\mathbf{q}}^{l} \beta_{\sigma} c_{\mathbf{k}+\mathbf{q}, l p \sigma}^{\dagger}\right)}{\left(E_{\mathbf{k}}-\varepsilon_{p}\right)}\right]|0\rangle,
\end{aligned}
$$

where $C$ is the normalization constant, $\alpha_{\uparrow} / \alpha_{\downarrow}=i \sqrt{S_{0}^{+} / S_{0}^{-}}$ $=i e^{i \eta}$, i.e., $\eta=\tan ^{-1} S_{0}^{y} / S_{0}^{x}$, and

$$
\frac{\beta_{\uparrow(\downarrow)}}{\alpha_{\uparrow(\downarrow)}} \approx \pm e^{i \xi}\left[1+\frac{\Sigma_{l}\left(\left|V_{\mathbf{k}}^{l}\right|^{2}-\left|V_{\mathbf{k}+\mathbf{q}}^{l}\right|^{2}\right)}{|\Sigma| U\left(E-\varepsilon_{p}\right)}\right],
$$

with $\xi=\arg (\Sigma)$. According to our calculation, the second term on the right-hand side of Eq. (8) does not contribute to the net dipole moment of the system. It does, however, give rise to dipole moment density waves (similar to chargedensity wave) of wave vector $2 \boldsymbol{q}$. Since it is not the focus of

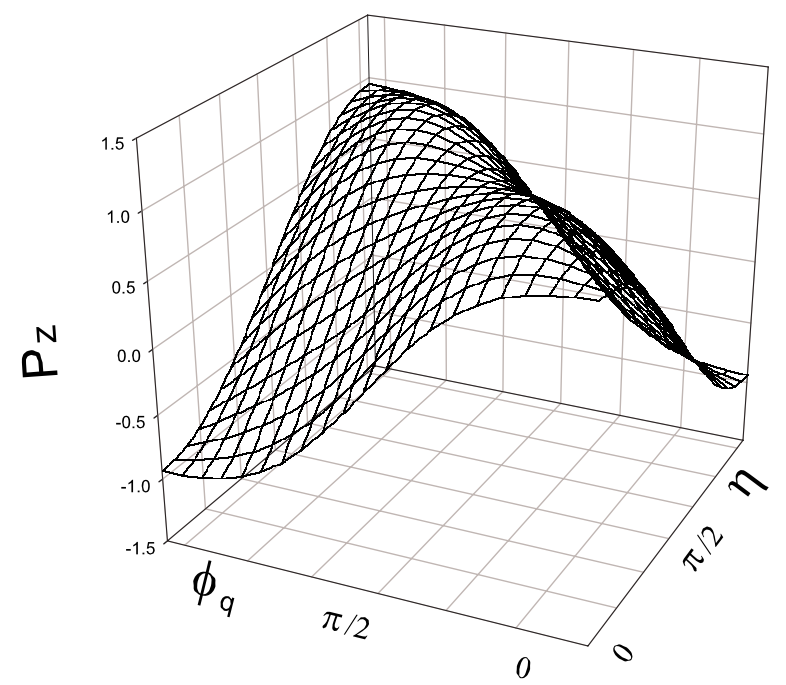

FIG. 1. The polarization in the $z$ direction vs $\eta$, the angle of spins on the $x y$ plane, and $\phi_{\mathbf{q}}$, the angle of $\mathbf{q}$ on the $x y$ plane. $\mathbf{P}_{z}$ is in arbitrary units. 
Brief Reports, we will drop it hereafter and use the relation

$$
\frac{\beta_{\uparrow(\downarrow)}}{\alpha_{\uparrow(\downarrow)}} \approx \pm e^{i \xi} .
$$

If $\gamma=0$ or the two magnetic orders are in phase and can be treated as one, then all the bands are twofold degenerate. This results in cancellation of electric dipole moment due to the difference in the relative sign between $\alpha_{\sigma}$ and $\beta_{\sigma}$ in two degenerate bands. In other words, if there is only one magnetic order $(\gamma=0)$, then there is no ferroelectricity. The following calculation is carried out under the assumption that $\gamma \neq 0$.

Anticipating the need of calculating the electric dipole moment per unit cell, we transform the wave function in Eq. (7) to a localized wave function

$$
\begin{aligned}
w_{j}(\boldsymbol{r})= & C \sum_{\sigma}\left(\alpha_{\sigma}+\beta_{\sigma} e^{\left.i \boldsymbol{q} \cdot \boldsymbol{R}_{j}\right)}\left\{\psi_{d \sigma}\left(\boldsymbol{r}-\boldsymbol{R}_{j}\right)+\sum_{l} \frac{V_{l}}{E_{0}-\varepsilon_{p}}\right.\right. \\
& \left.\times\left[\psi_{p l \sigma}\left(\boldsymbol{r}-\boldsymbol{R}_{j}-\boldsymbol{r}_{l}\right)-\psi_{p l \sigma}\left(\boldsymbol{r}-\boldsymbol{R}_{j}+\boldsymbol{r}_{l}\right)\right]\right\} \\
& -C \sum_{l, m, \sigma} \frac{V_{l}^{3} \cos \left(\boldsymbol{q} \cdot \boldsymbol{r}_{m}\right)}{2\left(E_{0}-\boldsymbol{\varepsilon}_{p}\right)^{3}}\left\{\left(\alpha_{\sigma} e^{i \boldsymbol{q} \cdot \boldsymbol{r}_{m}}+\beta_{\sigma} e^{i \boldsymbol{q} \cdot\left(\boldsymbol{R}-\boldsymbol{r}_{m}\right)}\right)\right. \\
& \times\left[\psi_{p l \sigma}\left(\boldsymbol{r}-\boldsymbol{R}_{j}+2 \boldsymbol{r}_{m}-\boldsymbol{r}_{l}\right)-\psi_{p l \sigma}\left(\boldsymbol{r}-\boldsymbol{R}_{j}+2 \boldsymbol{r}_{m}+\boldsymbol{r}_{l}\right)\right] \\
& +\left(\alpha_{\sigma} e^{\left.-i \boldsymbol{q} \cdot \boldsymbol{r}_{m}+\beta_{\sigma} e^{i \boldsymbol{q} \cdot\left(\boldsymbol{R}+\boldsymbol{r}_{m}\right)}\right)\left[\psi_{p l \sigma}\left(\boldsymbol{r}-\boldsymbol{R}_{j}-2 \boldsymbol{r}_{m}-\boldsymbol{r}_{l}\right)\right.}\right. \\
& \left.\left.-\psi_{p l \sigma}\left(\boldsymbol{r}-\boldsymbol{R}_{j}-2 \boldsymbol{r}_{m}+\boldsymbol{r}_{l}\right)\right]\right\},
\end{aligned}
$$

where subscript $\sigma$ is the spin index, $\psi_{p l \sigma}(\boldsymbol{r})$ is the wave function of the $p_{x(y)}$ orbital of the oxygen atoms, and $\psi_{d}(\boldsymbol{r})=A\left\langle\boldsymbol{r} \mid x^{2}-y^{2}\right\rangle+B\left\langle\boldsymbol{r} \mid 3 z^{2}-r^{2}\right\rangle$. Here, we have made the approximation $1 /\left(E_{\mathbf{k}}-\varepsilon_{p}\right) \approx 1 /\left(E_{0}-\varepsilon_{p}\right)$ $-\Sigma_{m}\left[V_{m} /\left(E_{0}-\boldsymbol{\varepsilon}_{p}\right)\right]^{2} \cos \left(\boldsymbol{q} \cdot \boldsymbol{r}_{m}\right) \cos \left[(2 \boldsymbol{k}+\boldsymbol{q}) \cdot \boldsymbol{r}_{m}\right]$, where $V_{l}$ $=\left(\Lambda_{l} A+B / \sqrt{3}\right)(p d \sigma)$ and $E_{0}=\varepsilon_{d}-J|\Sigma| / 2+\left(A^{2}+B^{2} / 3\right)$ $\times(p d \sigma)^{2} /\left(\varepsilon_{d}-J|\Sigma| / 2-\varepsilon_{p}\right)$ in Eq. (6).

The other interaction we considered was the spin-orbit coupling

$$
H_{1}=\lambda \boldsymbol{l} \cdot \boldsymbol{s} .
$$

Its effect can be seen in the following equations:

$$
\begin{gathered}
\boldsymbol{l} \cdot \boldsymbol{s}\left|x^{2}-y^{2}, \uparrow\right\rangle=i|x y, \uparrow\rangle+\frac{1}{2}(|z x, \downarrow\rangle-i|y z, \downarrow\rangle), \\
\boldsymbol{l} \cdot \boldsymbol{s}\left|x^{2}-y^{2}, \downarrow\right\rangle=-i|x y, \downarrow\rangle-\frac{1}{2}(|z x, \uparrow\rangle+i|y z, \uparrow\rangle), \\
\boldsymbol{l} \cdot \boldsymbol{s}\left|3 z^{2}-r^{2}, \uparrow\right\rangle=-\frac{\sqrt{3}}{2}(|z x, \downarrow\rangle+i|y z, \downarrow\rangle), \\
\boldsymbol{l} \cdot \boldsymbol{s}\left|3 z^{2}-r^{2}, \downarrow\right\rangle=\frac{\sqrt{3}}{2}(|z x, \uparrow\rangle-i|y z, \uparrow\rangle) .
\end{gathered}
$$

The appearance of $t_{2 g}$ orbitals demanded a little care. We defined the spin states parallel and antiparallel to $\boldsymbol{S}_{j}$ as

$$
|P\rangle_{j}=\cos \left(\theta_{j} / 2\right)|\uparrow\rangle_{j}+e^{i \phi_{j}} \sin \left(\theta_{j} / 2\right)|\downarrow\rangle_{j},
$$

$$
|A\rangle_{j}=-e^{-i \phi_{j}} \sin \left(\theta_{j} / 2\right)|\uparrow\rangle_{j}+\cos \left(\theta_{j} / 2\right)|\downarrow\rangle_{j},
$$

where $\theta_{j}$ and $\phi_{j}$ are the angles of $\boldsymbol{S}_{j}$. Since the states $|y z, P\rangle$, $|z x, P\rangle$, and $|x y, P\rangle$ are occupied by the $t_{2 g}$ electrons, we should project the spin states in Eq. (12) into antiparallel states. As a result, the spin-orbit coupling, when treated as a perturbation, modifies the $e_{g}$ orbitals in the following manner:

$$
\begin{aligned}
& \left|x^{2}-y^{2}, \uparrow\right\rangle_{j} \rightarrow\left|x^{2}-y^{2}, \uparrow\right\rangle_{j}+\frac{\lambda}{\Delta_{c f}-J(3+|\Sigma|) / 2} \\
& \times\left[-i e^{i \phi_{j}} \sin \frac{\theta_{j}}{2}|x y\rangle_{j}+\frac{1}{2} \cos \frac{\theta_{j}}{2}\left(|z x\rangle_{j}-i|y z\rangle_{j}\right)\right] \\
& \times|A\rangle_{j}, \\
& \left|x^{2}-y^{2}, \downarrow\right\rangle_{j} \rightarrow\left|x^{2}-y^{2}, \downarrow\right\rangle_{j}+\frac{\lambda}{\Delta_{c f}-J(3+|\Sigma|) / 2} \\
& \times\left[-i \cos \frac{\theta_{j}}{2}|x y\rangle_{j}+\frac{1}{2} e^{i \phi_{j}} \sin \frac{\theta_{j}}{2}\left(|z x\rangle_{j}+i|y z\rangle_{j}\right)\right] \\
& \times|A\rangle_{j}, \\
& \left|3 z^{2}-r^{2}, \uparrow\right\rangle_{j} \rightarrow\left|3 z^{2}-r^{2}, \uparrow\right\rangle_{j}-\frac{\lambda}{\Delta_{c f}-J(3+|\Sigma|) / 2} \frac{\sqrt{3}}{2} \\
& \times \cos \frac{\theta_{j}}{2}\left(|z x\rangle_{j}+i|y z\rangle_{j}\right)|A\rangle_{j} \\
& \left|3 z^{2}-r^{2}, \uparrow\right\rangle_{j} \rightarrow\left|3 z^{2}-r^{2}, \downarrow\right\rangle_{j}+\frac{\lambda}{\Delta_{c f}-J(3+|\Sigma|) / 2} \frac{\sqrt{3}}{2} e^{i \phi_{j}} \\
& \times \sin \frac{\theta_{j}}{2}\left(-|z x\rangle_{j}+i|y z\rangle_{j}\right)|A\rangle_{j},
\end{aligned}
$$

where $\Delta_{c f}$ is the crystal-field splitting between the $t_{2 g}$ and $e_{g}$ states. We inserted the forms in Eqs. (14) into Eq. (8).

Now, we are in a position to derive the electric polarization. The electric dipole moment of the $j$ th unit cell $\boldsymbol{P}_{i}$ is equal to $\int w_{j}^{*}(\boldsymbol{r}) \boldsymbol{r} w_{j}(\boldsymbol{r}) d^{3} \boldsymbol{r}$. Due to the symmetry of the atomic wave functions, the integrals that have to be considered are of the form $\int \psi_{d}^{*}\left(\boldsymbol{r}-\boldsymbol{R}_{j}\right) \boldsymbol{r} \psi_{p l}\left(\boldsymbol{r}-\boldsymbol{R}_{j} \pm \boldsymbol{r}_{l}\right) d^{3} \boldsymbol{r}$. It turns out that due to the symmetry of the lattice, all the terms with $\int \psi_{x^{2}-y^{2}}^{*}(\boldsymbol{r}$ $\left.-\boldsymbol{R}_{j}\right) \boldsymbol{r} \psi_{p l}\left(\boldsymbol{r}-\boldsymbol{R}_{j} \pm \boldsymbol{r}_{l}\right) d^{3} \boldsymbol{r}$ cancel out. What is left are the terms with $\int \psi_{x y}^{*}\left(\boldsymbol{r}-\boldsymbol{R}_{j}\right) \boldsymbol{r} \psi_{p l}\left(\boldsymbol{r}-\boldsymbol{R}_{j} \pm \boldsymbol{r}_{l}\right) d^{3} \boldsymbol{r}$, i.e., those states generated by the spin-orbit interaction with the $p$ orbitals of oxygen atoms. This is compatible with the concept of bondcentered ferroelectricity in Ref. 9. Here, we define $\boldsymbol{\rho}_{n, l}$ $=\int \psi_{n}^{*}(\mathbf{r}) \boldsymbol{r} \psi_{p l}\left(\mathbf{r} \pm \boldsymbol{r}_{l}\right) d^{3} \boldsymbol{r}$, where $n=y z, z x$, or $x y$, and our result is the following:

$$
\begin{aligned}
\boldsymbol{P}_{j}= & -2 e \sum_{l} \delta_{l}\left|C \alpha_{\uparrow}\right|^{2} \sin \left(2 \boldsymbol{q} \cdot \boldsymbol{r}_{l}\right)\left\{\sin \left(\boldsymbol{q} \cdot \boldsymbol{R}_{j}+\xi\right)\right. \\
& \times\left[(A-B) \sin \theta_{j} \cos \phi_{j} \boldsymbol{\rho}_{z x, l}-(A+B) \sin \theta_{j} \sin \phi_{j} \boldsymbol{\rho}_{y z, l}\right] \\
& +\cos \left(\boldsymbol{q} \cdot \boldsymbol{R}_{j}+\xi\right)\left[2 A \left(\cos \eta \sin \theta_{j} \sin \phi_{j}\right.\right. \\
& \left.-\sin \eta \sin \theta_{j} \cos \phi_{j}\right) \boldsymbol{\rho}_{x y, l}+(A-B) \cos \eta \cos \theta_{j} \boldsymbol{\rho}_{z x . l} \\
& \left.\left.-(A+B) \sin \eta \cos \theta_{j} \boldsymbol{\rho}_{y z, l}\right]\right\},
\end{aligned}
$$

where $\delta_{l}=\lambda V_{l}^{3} /\left(E_{0}-\varepsilon_{p}\right)^{3}\left[\Delta_{c f}-J(3+|\Sigma|) / 2\right]$. Note that $\boldsymbol{\rho}_{y z, x}$ $=\boldsymbol{\rho}_{z x, y}=0, \boldsymbol{\rho}_{y z, y}=\boldsymbol{\rho}_{z x, x}=\rho \hat{z}$, and $\boldsymbol{\rho}_{x y, x}=\rho \hat{y}, \boldsymbol{\rho}_{x y, y}=\rho \hat{x}$, with $\rho$ 
$=\int \psi_{z x(y)}^{*}(\boldsymbol{r}) z \psi_{p x(y)}\left(\boldsymbol{r} \pm \boldsymbol{r}_{x(y)}\right) d^{3} \boldsymbol{r}$. So the dipole moment is in the $z$ direction. Let us give an estimate of the magnitude of the resulting polarization. $\Delta_{c f} \approx 3 \mathrm{eV}, J \approx 2 \mathrm{eV},\left|\varepsilon_{d}-3 J / 2-\varepsilon_{p}\right|$ $\approx 2 \mathrm{eV},(p d \sigma) \approx 0.5 \mathrm{eV}$, and $\lambda \approx 0.05 \mathrm{eV}$. Since the volume of a unit cell is approximately $100 \AA^{3} / 2,\left|C \alpha_{\uparrow}\right|^{2} \approx 1 / 4$, and $\rho \approx 1 \AA, p_{z}$ is of the order $100 \mu \mathrm{C} / \mathrm{m}^{2}$, which is compatible with experimental data.

In order to see more clearly the meaning of Eq. (15), we considered a very simple case. Let $\gamma=\pi / 2$, and $A=1, B=0$ or $A=0, B=1$. These mean that the phase difference between the magnetic orders of $S^{z}$ and $S^{x(y)}$ is $\pi / 2$ and either $\left|x^{2}-y^{2}\right\rangle$ or $\left|3 z^{2}-r^{2}\right\rangle$ is considered. In Fig. 1, the resulting polarization $\mathbf{P}_{z}$ is plotted against the $\eta$ and $\phi_{\mathbf{q}}$, the angles between the $x$ axis and the projections of the spins and $\mathbf{q}$ on the $x y$ plane, respectively. $\mathbf{P}_{z}$ is in arbitrary units. It is interesting to note that the polarization is the largest if the difference between $\phi_{\mathbf{q}}$ and $\eta$ is equal to either 0 or $\pi$. In other words, the projections of spins and $\mathbf{q}$ on the $x y$ plane are collinear. We can simplify the matter further by assuming $\eta=0$ and $S_{0}^{y}=0$. Then Eq. (15) is simplified to

$$
\begin{aligned}
\mathbf{P}_{j} \simeq & \frac{e \rho \lambda}{S_{0}^{z} S_{0}^{x}\left[\Delta_{c f}-J(3+|\Sigma|) / 2\right]}\left[\frac{(p d \sigma)}{E_{0}-\varepsilon_{p}}\right]^{3}\left[\left(S_{j+x}^{z}-S_{j-x}^{z}\right) S_{j}^{x}\right. \\
& \left.-\left(S_{j+x}^{x}-S_{j-x}^{x}\right) S_{j}^{z}\right] \hat{z},
\end{aligned}
$$

where $\boldsymbol{S}_{j+x(y)}$ is the spin at $\boldsymbol{R}_{j} \pm 2 \boldsymbol{r}_{x(y)}$. We can rewrite Eq. (16) to give a compact expression of the electric polarization $\boldsymbol{p} \sim \hat{z}\left[M_{z} \boldsymbol{\nabla} \cdot \boldsymbol{M}-(\boldsymbol{M} \cdot \boldsymbol{\nabla}) M_{z}\right]$. The phenomenological model ${ }^{14}$ gives a similar form: $\boldsymbol{p} \sim[\boldsymbol{M}(\nabla \cdot \boldsymbol{M})-(\boldsymbol{M} \cdot \nabla) \boldsymbol{M}]$. On the one hand, what we have calculated is simpler. Only the dynamics on the $a b$ plane was considered and this is the reason why the polarization is pointing in the $z$ direction. We must add that this approximation is compatible with the structures of the systems such as $\mathrm{TbMnO}_{3}$. On the other hand, there is significant complication in our calculation, namely, the dynamics and anisotropy of orbitals. It is remarkable that we reached the expression of Ref. 14. There had been a long history ${ }^{1}$ of studying the ferroelectricity phase transition with Landau's theory. If Mostovoy's model, ${ }^{14}$ which is supported by our calculation, is correct, then there should be a term of the form $g \boldsymbol{p} \cdot[\boldsymbol{M}(\nabla \cdot \boldsymbol{M})-(\boldsymbol{M} \cdot \nabla) \boldsymbol{M}]$ in the thermodynamic potential where $g$ is a constant. This implies that the phase transition is an improper ferroelectric phase transition ${ }^{15,16}$ and the magnetic orders, instead of the electric polarization order, should be the order parameters. All the thermody- namic properties should be understood thusly.

As for the mechanism, the spin-current calculation proposed by Katsura et al. ${ }^{10}$ gave an electric dipole moment of the form $\hat{e} \times\left(\boldsymbol{S}_{1} \times \boldsymbol{S}_{2}\right)$, with $\hat{e}$ being the direction of the $M-\mathrm{O}-M$ bond. This is equivalent to our result in Eqs. (15) and (16) and Mostovoy's form ${ }^{14}$ if the expressions in Eqs. (2) are used. If there is only one sinusoidal magnetic order, then there is no spin current. Our calculation, indeed, showed that if there is no phase difference between the magnetic orders in the $x(y)$ direction and $z$ direction, there is no electric polarization. It is because, in this case, all the magnetic orders can be combined into one sinusoidal order. This is consistent with the experimental finding. ${ }^{6}$ In the temperature range between 41 and $28 \mathrm{~K}$, there is one sinusoidal magnetic order but no net electric polarization in $\mathrm{TbMnO}_{3}$. Even though Katsura et al. ${ }^{10}$ considered $t_{2 g}$ orbitals and their $\pi$ bonds with oxygen atoms and two noncollinear spins, while we considered $e_{g}$ orbitals, $\sigma$ bonds, and magnetic orders, the spin-current interpretation certainly accord with our calculation.

The present treatment considers the $e_{g}$ orbitals which are closest to the Fermi level, and thus play the essential role of determining physical properties for manganites. In particular, the crucial Hund's coupling term is properly treated. Hence it is a more suitable model for describing orthorhombic perovskite manganites than Ref. 10. Another important progress made by the current work is that the coupling between electric polarization and magnetic orders was considered instead of a small cluster of atoms. It is not only more physical but also provides an interesting connection [Eq. (16)] between the microscopic approach and Landau's theory of phase transition. ${ }^{14-16}$ The complete result [Eq. (15)] is much more complicated compared to Ref. 14, as the latter phenomenological model cannot account for the symmetry of orbitals and the detailed crystal structures. In conclusion, we have shown that there is, indeed, a direct relationship between magnetic orders and ferroelectricity, with the spin-orbit interaction being the link and the anisotropy of orbitals as the hotbed of dipole moments.

The author is indebted to Chung-Yu Mou and Di-Jing Huang for inspiring discussion, and benefitted from the activities of SCES and "spin-related physics in condensed matter" focus groups of NCTS, Taiwan. This work is supported in part by the National Science Council under Contract No. NSC 94-2112-M-002-46.
${ }^{1}$ For a review of earlier development, see G. A. Smolenskii and I. E. Chupis, Sov. Phys. Usp. 25, 475 (1982).

${ }^{2}$ Z. J. Huang et al., Phys. Rev. B 56, 2623 (1997).

${ }^{3}$ B. Lorentz et al., Phys. Rev. B 70, 212412 (2004).

${ }^{4}$ T. Kimura et al., Phys. Rev. B 71, 224425 (2005).

${ }^{5}$ T. Kimura et al., Nature (London) 426, 55 (2003).

${ }^{6}$ M. Kenzelmann et al., Phys. Rev. Lett. 95, 087206 (2005).

${ }^{7}$ Chong Gui Zhong and Qing Jiang, J. Phys.: Condens. Matter 14, 8605 (2002).

${ }^{8}$ I. A. Sergienko and E. Dagotto, Phys. Rev. B 73, 094434 (2006).
${ }^{9}$ Dmitry Efremov et al., Nat. Mater. 3, 853 (2004).

${ }^{10}$ Hosho Katsura et al., Phys. Rev. Lett. 95, 057205 (2005).

${ }^{11}$ I. Dzyaloshinskii, J. Phys. Chem. Solids 4, 241 (1958).

${ }^{12}$ T. Moriya, Phys. Rev. 120, 91 (1960).

${ }^{13}$ J. C. Slater and G. F. Koster, Phys. Rev. 94, 1498 (1954).

${ }^{14}$ Maxim Mostovoy, Phys. Rev. Lett. 96, 067601 (2005).

${ }^{15}$ A. P. Levanyuk and D. G. Sannikov, Sov. Phys. Usp. 17, 199 (1974).

${ }^{16}$ V. L. Indenboom, Sov. Phys. Crystallogr. 5, 106 (1960). 